\title{
On Some New Results of Poverty Orderings and Their Applications
}

\author{
Mervat Mahdy \\ Department of Statistics, Mathematics and Insurance, \\ Benha University, Egypt \\ drmervat.mahdy@fcom.bu.edu.eg
}

Received 6 June 2014

Accepted 2 September 2016

\begin{abstract}
The paper proposes to derive some new poverty indices which depend on aging classes. We also give some properties of it and show the connection between economic measure and new poverty measures these based on the concept of reversed residual incomes. In addition, the characterization of Pareto distribution based on new poverty functions is obtained. Furthermore, the stochastic orderings of new poverty indices are studied and their properties. In addition, the weighted poverty gap indices and stochastic dominance which involve the concept of inactivity incomes and its features are studied.
\end{abstract}

Keywords: Poverty gap; the severity of poverty; poverty ordering; weighed functions; lorenz curve; the reversed proportional failure rate.

2000 Mathematics Subject Classification: 60E15

\section{Introduction}

Let $X \geq 0$ be the random variable expresses the income of individuals with a density function $f_{X}(\theta)$, a distribution function $\left(F_{X}(\theta)\right)$, a reversed hazard rate $\widetilde{r}_{X}(\theta)=f_{X}(\theta) / F_{X}(\theta)(R H)$ and a poverty line $\theta$, where $\theta \in \mathfrak{R}^{+}$are considered; such that $F_{X}(\theta)$ represents the proportion of the poor. The poverty is then quantified in term of the proportion of the poor people and their income inequality (see Atkinson [1], Foster and Shorrocks [2,3] and Belzunce et al. [4]).

The difference between the poverty line $\theta$ and individual income $y_{i}$ is defined as the poverty gap, $P_{i}$, where $P_{i}=\theta-y_{i}$. The traditional poverty gap index is introduced by Sen [5] as the percentage of individuals below $\theta$. Almost the poverty papers consider the poverty gap index as

$$
\Delta=\sum_{i \in \aleph} P_{i} /(m \theta), \aleph \text { is the set of poor individuals. }
$$

it depends on uniform distribution. In many cases, the distribution of income is not uniform distribution.

There are some related aging classes which related to $\widetilde{r}_{X}(\theta)$ such as mean reversed hazard lifetime. It should be noted that the $\widetilde{r}_{X}(\theta)$ function and its related functions are less intuitive functions.

\footnotetext{
* Tel: +20-12-068-2460/ +20-22-840-6099, fax: +20-13-323-0860.
} 
The first goal of this study is to introduce new poverty indices which depend on distribution function of income in general cases, i.e., the distribution of income may be not uniform such as exponential or Pareto distribution and so on. Moreover, these indices consider as right truncated distribution. In addition, the second goal of this paper is spread out the usefulness of $\widetilde{r}_{X}(\theta)$ by relating it to new poverty gap indices.

The study of the poverty measures (poverty gap and the poverty severity) based on the concept of reversed residual incomes are defined in Section 2. We also give some properties of it and show the relationship between Lorenz curve and new poverty measures these based on the concept of reversed residual incomes. In addition, the characterization of Pareto distribution based on new poverty functions is obtained. Section 3 consists of a through the study of the stochastic dominance of new poverty measures. The last section provides the weighted poverty gap function and stochastic dominance which involve the concept of inactivity incomes and its features are studied.

\section{The Poverty Measures Functions}

Let $X \geq 0$ be the random variable expresses the income of individuals with an absolutely continuous distribution function $F_{X}($.$) , and a density function f_{X}($.$) . We consider F_{X}(\theta)$ for a poverty line $\theta$ as a right truncated income distribution at $\theta$, then we interesting to study the random variable:

and its distribution function is given by

$$
X_{r}(\theta)=\{X \mid X \leq \theta\}
$$

$$
F_{X_{r}(\theta)}(x)=\frac{F_{X}(x)}{F_{X}(\theta)} \text { for } x \leq \theta .
$$

Then, the mean of $x_{r}(\theta)$ (the average income below the poverty line $\theta$ ) can define as follows:

$$
\psi(\theta)=E(X \mid X \leq \theta)=\int_{0}^{\theta} s d F_{X}(s) / F_{X}(\theta), \quad \theta \in \mathfrak{R}^{+} .
$$

It is satisfies the following properties:

- $\psi(\theta) \leq \theta$, for all $\theta \in \mathfrak{R}^{+}$,

- $\lim _{\theta \rightarrow \infty} \psi(\theta)=E(\theta)$.

and its distribution function is given by

The following definition is essential for this work:

Definition 2.1: Let the random variable $X$ expresses the income of individuals, with distribution function $F_{X}($.$) and a density function f_{X}($.$) . Then, the reversed proportional failure rate (\mathrm{RPF}), \widetilde{p}_{X}(\theta)$, is defined as follows:

$$
\widetilde{p}_{F}(\theta)=\theta f_{X} / F_{X}, \text { for all } \theta \in \mathfrak{R}^{+} .
$$

The reversed proportional failure rate function has been studied by Block et al. [6], Chandra and Roy [7], Finkelstein $[8,9,10]$ and Gupta et al. [11].

Then, by using (2.1), we can obtain the poverty gap (PG) index as the following formula

Moreover, from (2.2) and (2.3), we obtain:

$$
\beta_{X}(\theta)=1-\psi(\theta) / \theta, \quad \text { for all } \theta \in \mathfrak{R}^{+} .
$$

$$
\partial \beta_{X}(\theta) / \partial \theta=(1 / \theta)\left[1-\widetilde{p}_{X}(\theta) \beta_{X}(\theta)\right], \text { for all } \theta \in \mathfrak{R}^{+},
$$

where $\widetilde{p}_{X}(\theta)$ is the reversed proportional failure rate function at a poverty line $\theta$.

Moreover, the relationship between $\psi(\theta)$ and $\beta_{X}(\theta)$ is given by

$$
\psi(\theta)=\theta\left[1-\beta_{X}(\theta)\right]
$$


and

$$
\partial \psi(\theta) / \partial \theta=\widetilde{p}_{X}(\theta) \beta_{X}(\theta), \text { for all } \theta \in \mathfrak{R}^{+} .
$$

In the current investigation, we study the properties of new poverty index in term of axioms for a good index of poverty.

Theorem 2.1: The poverty gap index of (2.3) satisfying axioms:

- Normalization (N),

- Increasing in subsistence income (ISI),

- Monotonicity (M).

Proof. It is easy to proof that if all incomes have zero. Therefore, $\beta_{X}(\theta)$ takes the value unity, it is means that $\beta_{X}(\theta)$ is satisfying $N$. For proof that $\beta_{X}(\theta)$ is increasing in $\theta$, we should be achieve the following relation

$$
\widetilde{p}_{X}(\theta) \beta_{X}(\theta)<1 \text {, }
$$

since $E(X \mid X \leq \theta)<\theta \Rightarrow E(X \mid X \leq \theta) / \theta<1$ and $\widetilde{p}_{X}(\theta)$ is probability value, then $\widetilde{p}_{X}(\theta) \beta_{X}(\theta)<1$. This means that $\beta_{X}(\theta)$ is satisfying $I S I$. When we reduction in an income with value $a$ below $\theta$, the poverty gap index can be shown as

since $a$ and $\theta$ are positive numbers, then

$$
\begin{aligned}
\beta_{X-a}(\theta) & =1-\left(\int_{0}^{\theta} x d F_{X}(x) / F_{X}(\theta)-a\right) / \theta, \text { for all } \theta \in \mathfrak{R}^{+} \\
& =1-(\psi(\theta) / \theta)+(a / \theta)=\beta_{X}(\theta)+(a / \theta),
\end{aligned}
$$

$$
\partial \beta_{X-a}(\theta) / \partial \theta \geq \partial \beta_{X}(\theta) / \partial \theta, \text { for all } \theta \in \mathfrak{R}^{+},
$$

it is complete proof that $\beta_{X}(\theta)$ is satisfying $M$.

To compare the distribution of income of different countries at the same time we may be used lorenz curve that is introduced by Lorenz [12], and it is also, defined by Gastwirth [13] as following definition:

Definition 2.2: Let $W \geq 0$ be a random variable with cumulative distribution function $F_{W}(\theta)$ and a density function $f_{W}(\theta)$, with a finite mean $\mu$. The Lorenz curve $L_{W}(p)$ of $W$ is defined in terms of two parametric equations in $x$ namely

$$
p=F_{W}(\theta)=\int_{0}^{\theta} d F_{W}(t)
$$

and

$$
L_{w}(\theta)=F_{1}(\theta)=\frac{1}{\mu} \int_{0}^{\theta} t f_{W}(t) d t,
$$

where $F_{W}(\theta)$ can interpret as the proportion of the poor below the level $\theta \cdot F_{1}(\theta)$ can be viewed as the proportional share of the total income of poor below the level $\theta$.

It follows from (2.4) that the Lorenz curve is the first moment distribution function of $F_{W}(\theta)$. It may be noticed that both $F_{W}(\theta)$ and $F_{1}(\theta)$ lies between zero and one. In addition, the Lorenz curve being the plot of the points $\left(F_{W}(\theta), F_{1}(\theta)\right)$ is represented in the unit square. $L_{W}(p)$ can be interpreted as the proportion of the total wealth possessed by the poorest $p^{\text {th }}$ fraction of the population. The Lorenz curve defined by (2.4) acquires the following properties:

- $L(0)=0, L(1)=1$, and $L_{W}(p)$ is continuous and strictly increasing on $(0,1)$, as

$$
\partial L_{W}(p) / \partial \theta=\theta(F) / \mu \text {, for all } \theta \in \mathfrak{R}^{+} .
$$


where

$$
L_{W}(p)=\int_{0}^{p} x\left(F_{1}\right) d F_{1} / \int_{0}^{1} x\left(F_{1}\right) d F_{1} \text { and } x\left(F_{1}\right)=\inf \left\{y: F(y) \geq F_{1}\right\} .
$$

- $\quad L_{W}(p)$ is twice differentiable and is strictly convex on $(0,1)$ as

$$
\partial^{2} L_{W}(p) / \partial \theta^{2}=1 / \mu f(\theta(F) w)>0 .
$$

where the inverse of the distribution function can interpret as $x(F)$.

For $W \geq 0$ be any random variable with distribution function $F_{W}$ and a finite mean $\mu$, the Lorenz curve $L_{W}(p)$ is defined as

$$
L_{w}(p)=\frac{1}{\mu} \int_{0}^{p} F_{W}^{-1}(t) d t, \text { for all } p \in[0,1],
$$

where $F_{\theta}^{-1}(t)=\inf \left\{\theta: F_{W}(\theta) \geq t\right\}$ is the left continuous inverse of $F_{W}$ (also known as the quantile function).

Thompson [14] has proved the following properties for the Lorenz curve defined by (2.5).

We can use the following characterizations theorem for the Pareto distribution (Pareto(.)) by using new measures.

Theorem 2.2: Suppose $W$ be a non-negative random variable. Then we get

1.The poverty gap function is

$$
\beta_{W}(\theta)=1-\frac{\alpha^{\kappa} \kappa \theta^{1-\kappa}}{(1-\kappa) p} .
$$

2. If $r_{W}(\theta)=f_{W}(\theta) /\left(1-F_{W}(\theta)\right)$ represents the hazard rate and $\beta_{W}(\theta)$ the poverty gap function, therefore, the relationship

$$
\beta_{W}(\theta)=1-\frac{\theta^{2}}{(1-\kappa)} r_{W}(\theta) .
$$

3. If $W$ have the Lorenz curve $L_{W}(p)$, the poverty gap $\beta_{W}(\theta)$ and the finite mean $\mu$, then the relationship

4.We have the following relationship

$$
\beta_{W}(\theta)=1-g_{1}(\Theta) L_{W}(\theta) / \mu ; \quad g_{1}(x)=1 / p
$$

$$
\beta_{W}(\theta)=1+\frac{\theta\left(1-L_{W}(p)\right)}{p L_{W}(p)},
$$

where $L_{W}(p)=\partial L_{W}(p) / \partial p$. Will hold for all real $p \geq 0$ if and only if $W$ follows Pareto $(\kappa, \alpha)$ with distribution function as follows:

$$
F_{W}(\theta)=1-(\alpha / \theta)^{\kappa}, \quad \theta \geq \alpha \text { and } \kappa>1 .
$$

Proof. Since

$$
\psi(\theta)=\frac{\alpha^{\kappa} \kappa \theta^{1-\kappa}}{(1-\kappa)\left(1-\alpha^{\kappa} \theta^{-\kappa}\right)}=\frac{\alpha^{\kappa} \kappa \theta^{1-\kappa}}{(1-\kappa) p}, \text { for all } \theta \in \mathfrak{R}^{+} ; \kappa \neq 0 .
$$

Hence by (2.3) the required result (1) follows.

In addition, when $r_{W}(\theta)$ holds. Hence, we can calculate $\beta_{W}(\theta)$ directly by using (2.6) as in Eq. (2.7).

Furthermore,

$$
L_{W}(\theta)=\frac{1}{\mu} \int_{0}^{\theta} t d F_{W}(t) d t=\alpha^{\kappa-1} \frac{\theta^{1-\kappa}}{(1-\kappa)} \text { and } \mu=\kappa \theta .
$$

Then the required result (3) follows.

Assume Eq. (2.9) holds, and according to Arnold [15], we obtain: 
and

$$
L_{W}(s)=1-(1-s)^{-1 / \kappa+1}
$$

$$
\partial L_{W}(s) / \partial s=(\kappa-1) /\left(\kappa(1-s)^{1 / \kappa}\right), \text { forall } \kappa>1 .
$$

Hence, (2.6) gives the needed result (3).

Next, we consider a new measure of the poverty that takes the variations in the distribution of welfare amongst the poor into account. It is defined as the severity of poverty.

Let $X \geq 0$ be a random variable denote an income below level $\theta$ with cumulative distribution function $F_{X}($.). In many economic problems, there are attention to the following random variable:

$$
X_{r}^{2}(w)=\left\{X^{2} \mid X \leq \theta\right\} .
$$

The severity of the poverty (SP) of the poor people is defined as

$$
\rho_{X}(\theta)=E\left(\left(\frac{\theta-X}{\theta}\right)^{2} \mid X \leq \theta\right) .
$$

By using integration by parts, we can conclude that:

$$
\rho_{X}(\theta)=\frac{2}{\theta^{2} F_{X}(\theta)} \int_{0}^{\theta} \int_{0}^{y} F_{X}(x) d x d y=\frac{2}{\theta}\left[\theta \beta_{X}(\theta)-\Phi(\theta)\right], \text { for all } \theta \in \mathfrak{R}^{+},
$$

where

$$
\Phi(\theta)=\int_{0}^{\theta} x F_{X}(x) d x / \theta F_{X}(\theta) .
$$

Now, we can define new class of the severity as follows:

A random variable $X$ is said to have increasing (decreasing) the poverty severity, IPS (DPS), if

$$
\rho_{X}(\theta) \text { is increasing (decreasing) in } \theta,
$$

or, equivalently, $F_{X} \in I P S(D P S)$ iff

$$
\beta_{X}(\theta) \geq(\leq) \Phi(\theta)
$$

\section{Stochastic Dominance of The Poverty Measures Functions}

Let $X \geq 0$ and $Y \geq 0$ have the distribution functions $F$ and $G$, and the reversed proportional failure rate $(R P F)$ functions $\widetilde{r}$ and $\widetilde{q}$ respectively. Then $X$ is said to be smaller than or equal $(\leq) Y$ in $R P F$ order (denoted as $\left.X \leq_{r p} Y\right)$ if,

$$
\widetilde{r}(\theta) \leq \widetilde{q}(\theta), \text { for all } \theta \in \mathfrak{R}^{+} .
$$

To compare the poverty gap function of two group poor people, let $X$ and $Y$ be two nonnegative random variables (incomes of two poor groups), having distribution functions $F_{X}$ and $G_{X}$, and the poverty gap functions $\beta_{X}(\theta)$ and $\beta_{Y}(\theta)$ respectively. The following definition of the poverty gap ordering is essential for this section:

Definition 3.1: $X$ is said to be smaller than or equal to $Y$ in the poverty gap function ordering $\left(X \leq_{p g} Y\right)$ if

$$
\beta_{X}(\theta) \geq \beta_{Y}(\theta), \quad \text { for all } \theta \geq 0,
$$

where $\theta$ is a poverty line. It can be written as 


$$
\int_{0}^{\theta} F_{X}(u) G_{Y}(\theta) d u \geq \int_{0}^{\theta} G_{Y}(u) F_{X}(\theta) d u, \quad \text { for all } \theta \in \mathfrak{R}^{+} .
$$

There is important notice that reversed mean residual lifetime ordering is same of new poverty index, that is introduced in above definition where, if we suppose $m_{F}(x)=\int_{-\infty}^{x} F_{X}(u) / F_{X}(x) d u$ is reversed mean residual lifetime, we get that $\beta_{X}(\theta)=m_{F}(\theta) / \theta$. Now, by using Theorem (2.2) in Ahmad et al. [16] and Corollary (3.1) and Theorems (3.2 and 3.3) in Kayid and Ahmad [17] we can conclude that:

(i). If $U_{1}, U_{2}$ and $Z \geq 0$ be three nonnegative random variables, where $Z$ is independent of both $U_{1}$ and $U_{2}$ and let $Z$ have a density $g$. If $U_{1} \leq_{p g} U_{2}$ and $g$ is a log-concave that is $Z$ has the decreasing the reversed proportional failure rate property, then we can get that $U_{1}+Z \leq_{p g} U_{2}+Z$.

(ii). If $U_{1} \leq_{p g} Z_{1}$, and $U_{2} \leq_{p g} Z_{2}$, also $U_{1}$ is independent of $U_{2}$ and $Z_{1}$ is independent of $Z_{2}$, then the following statements holds:

(a) If $U_{1}$ and $Z_{2}$ have log-concave densities, then $U_{1}+U_{2} \leq_{p g} Z_{1}+Z_{2}$.

(b) If $U_{2}$ and $Z_{1}$ have log-concave densities, then $U_{1}+U_{2} \leq_{p g} Z_{1}+Z_{2}$.

(iii). If $U_{1}, U_{2}, \ldots$, and $Z_{1}, Z_{2}, \ldots$, are sequences of independent random variables with $U_{i} \leq_{p g} Z_{i}$ and $U_{i}$ and $Z_{i}$ have log-concave densities for all $i$, then

$$
\sum_{i=1}^{n} U_{i} \leq_{p g} \sum_{i=1}^{n} Z_{i}, \quad n=1,2, \ldots
$$

According (3.1), we can say that the poverty gap order is closed under convolution.

(iv). Suppose that $U(\varsigma)$ be a random variable having distribution function $F_{\varsigma}$ and let $\Theta_{i}$ be a random variable having distribution $G_{i}$, for $i=1,2$. Let $U(\varsigma), \varsigma \in \mathfrak{R}^{+}$be a family of a random variables independent of $\Theta_{1}$ and $\Theta_{2}$. If

$$
U\left(\varsigma_{1}\right) \leq_{p g} U\left(\varsigma_{2}\right) \text {, for all } \varsigma_{1} \leq \varsigma_{2},
$$

then

$$
U\left(\Theta_{1}\right) \leq_{p g} U\left(\Theta_{2}\right) .
$$

According (3.2), we can say that $\leq_{p g}$ is closed under mixture.

The lower and higher bounds for mixture weights of two distributions by using RPF ordered are studied in the next result.

Theorem 3.1: Suppose $U \geq 0$ and $V \geq 0$ be two random variables with distribution functions $K($.$) and M($. respectively. Let $Z$ is mixture of $U$ and $V$ with distribution $H()=.A K()+.(1-A) M(),. \forall A \in(0,1)$. Then we have $U \leq_{r p} Z \leq_{r p} V$ if $U \leq_{r p} V$.

Proof: If $U \leq_{r p} V$, then we have

$$
\frac{M(.)}{K(.)} \text { is increasing in } \theta \text {. }
$$

Also, let $u_{U}, u_{V}$ and $u_{Z}$ denote the right endpoints of the supports of the corresponding random variables $U, V$ and $Z$ respectively, and $\max \left(u_{U}, u_{Z}\right)=\max \left(u_{U}, u_{V}\right)$, then

$$
\frac{A K(\theta)+(1-A) M(\theta)}{K(\theta)}=A+(1-A) \frac{M(\theta)}{K(\theta)},
$$


is increasing in $\theta \in\left(-\infty, \max \left(u_{U}, u_{Z}\right)\right)$. Therefore, by (3.3), we can get that $U \leq_{r p} Z$. The proof that $Z \leq_{r p} V$ is similar.

Next, we define a new ordering of random variables which depend on the poverty severity function that is the poverty severity order.

Let $X$ and $Y$ be random variables with distribution function $K($.$) and M($.$) and S P$ functions $\rho_{K}(\theta)$ and $\rho_{M}(\theta)$, respectively. Then $X$ is said to be smaller than $Y$ in the poverty severity ordering, $X \leq_{p s} Y$, if

$$
\int_{0}^{\theta} \int_{0}^{y} K(x) d x d y / K(\theta) \leq \int_{0}^{\theta} \int_{0}^{y} M(x) d x d y / M(\theta), \quad \text { for all } \theta \in \mathfrak{R}^{+},
$$

equation (3.4) can equivalently be written as

In addition, Eq. (3.5) is equivalent

$$
\frac{\int_{0}^{\theta} \int_{0}^{y} K(x) d x d y}{\int_{0}^{\theta} \int_{0}^{y} M(x) d x d y}, \quad \text { is increasing in } \theta \in \mathfrak{R}^{+} .
$$

$$
\rho_{K}(\theta) \leq \rho_{M}(\theta) \text {, for all } \theta \in \mathfrak{R}^{+} .
$$

\section{The Weighted Poverty Gap}

Among the key causes, or at least correlates, of poverty is hours worked $(H)$, according to Borjas [18] we can write the relationship between income variable $(X)$ and hours worked variable $(\mathrm{H})$ as follows:

$$
H_{F}(x)=4 \exp (\alpha+\beta \ln x) \text {, for all } x \in \mathfrak{R}^{+} .
$$

If we need to take into account the hours worked variable during measure the poverty index, we can use this variable as weighted function $\left(H_{F}(x)\right)$. Let the observation, $x$, of $X$ is recorded with $H_{F}(x)$. Then $x$ is an observation on the weighted random variable, $X^{H}$, with pdf function as follows:

$$
\begin{aligned}
& f^{H}(x)=\frac{H_{F}(x) f(x)}{E\left(H_{F}(x) \mid x \leq \theta\right)} \\
& =\frac{4 \exp (\alpha+\beta \ln x) f(x)}{E(4 \exp (\alpha+\beta \ln x) \mid X \leq \theta)}, \text { for all } x \in \mathfrak{R}^{+} .
\end{aligned}
$$

where $E\left(H_{F}(x) \mid x \leq \theta\right)>0, \alpha$ and $\beta$ are parameters and $\theta$ is poverty line. The random variable $X^{H}$ is called the weighted version of $X$, and its distribution is:

$$
F^{H}(x)=\frac{F(x)\left[H_{F}(x)-A_{F}(x)\right]}{E(4 \exp (\alpha+\beta \ln x) \mid X \leq \theta)},
$$

where

$$
A_{F}(y)=\int_{0}^{y} \frac{H_{F}^{\backslash}(r) F(r) d r}{F(y)}=\int_{0}^{y} \frac{4 \beta \exp (\alpha+\beta \ln r) F(r) d r}{r F(y)} .
$$

where $H_{F}^{\backslash}(x)=\partial H_{F}(x)$. Note that if $H_{F}^{\backslash}(x)>0$, then $A_{F}(u) \geq 0 \forall u \in \mathfrak{R}^{+}$.

Now, we can be defined

$$
B_{F}(s)=E\left[H_{F}(s) \mid X \leq s\right]=\int_{0}^{s} \frac{H_{F}(x) f(x) d x}{F(s)}=\int_{0}^{s} \frac{4 \exp (\alpha+\beta \ln x) f(x) d x}{F(s)} .
$$

Then (4.2) can be rewritten as the following

Moreover, if

$$
F^{H}(s)=\frac{F(s) B_{F}(s)}{E\left(H_{F}(s) \mid s \leq \theta\right)},
$$




$$
D_{F}(s)=E\left[H_{F}(s) \mid X>s\right]=\int_{s}^{\infty} \frac{H_{F}(y) f(y) d y}{\bar{F}(s)}=\int_{0}^{s} \frac{4 \exp (\alpha+\beta \ln x) f(x) d x}{F(s)} .
$$

Reintroducing $E\left(H_{F}(s) \mid s \leq \theta\right)$ into (4.4), it follows

$$
E\left(H_{F}(s) \mid s \leq \theta\right)=B_{F}(s) F(s)+D_{F}(s) \bar{F}(s) .
$$

By using (2.2), then RPF of $X^{H}$ can rewrite as

$$
\widetilde{p}_{X^{H}}(s)=H_{F}(s) \tilde{p}_{X}(s) / B_{F}(s) .
$$

Now, suppose we use $H_{F}(x)$ as the rate of increase in equality per pound squared, as income is transmitted through income level $x$ from poorer to richer individuals (see for more details, Di Crescenzo [19], Solon [20], Barrett and Salles [21] and Ichino et al. [22]). Also, by using (2.1), (4.2) and (4.4) we have the weight PG function $\left.\beta_{X^{H}}(\theta)\right)$ of $F^{H}(x)$ as follows

$$
\beta_{X^{H}}(\theta)=\frac{\int_{0}^{\theta} F(y)\left[H_{F}(y)-A_{F}(y)\right] d y}{\theta F(\theta)\left[H_{F}(\theta)-A_{F}(\theta)\right]}=\frac{\int_{0}^{\theta} F(y) B_{F}(y) d y}{\theta F(\theta) B_{F}(\theta)} .
$$

In the following examples, we illustrate the weighted poverty gap functions for some important income distributions.

Example 4.1 (The Pareto II distribution). The Pareto II distribution is important for studying the growth rate of income poor. Lomax [23] suggested pdf of the Pareto II distribution as:

$$
g_{X}(\theta)=\xi(1+\xi \theta)^{-1}, \text { for all } \theta, \xi \in \mathfrak{R}^{+} .
$$

In addition, the cumulative distribution function of $g_{X}(\theta)$ is follows:

$$
G_{X}(\theta)=1-(1+\xi \theta)^{-1} \text {, for all } \theta, \xi \in \mathfrak{R}^{+} .
$$

Given (4.2), after some algebraic calculations, we obtain

$$
\begin{aligned}
E(4 \exp (\alpha+\beta \ln x) \mid X \leq \theta) & =\int_{0}^{\theta} \xi \exp (\alpha+\beta \ln u)(1+\xi u)^{-2} d u . \\
& =\xi \exp (\alpha) U(\theta ; k, \xi, \beta),
\end{aligned}
$$

and

$$
\begin{aligned}
A_{G}(\theta) & =\int_{0}^{\theta} \frac{4 \beta \exp (\alpha+\beta \ln u)\left(1-(1+\xi u)^{-1}\right) d u}{u\left(1-(1+\xi \theta)^{-1}\right)}, \\
& =\frac{4 \beta \exp (\alpha)}{1-(1+\xi \theta)^{-1}} V(\theta ; k, \xi, \beta) .
\end{aligned}
$$

By using (4.2), we can be concluded the weighted cumulative distribution function is given by

where

$$
\begin{aligned}
& G^{H}(x)=\frac{G_{X}(x)\left[H_{G}(x)-A_{G}(x)\right]}{E(4 \exp (\alpha+\beta \ln x) \mid X \leq \theta)}, \\
& =\frac{4 \theta^{\beta} G_{X}(\theta)-4 \beta V(\theta ; k, \xi, \beta)}{\xi U(\theta ; k, \xi, \beta)},
\end{aligned}
$$

$$
\begin{aligned}
& U(x ; k, \xi, \beta)=\sum_{k=1}^{\beta-1}(-1)^{k-1} \frac{k x^{\beta-k}}{(\beta-k) \xi^{k+1}}+\frac{(-1)^{\beta-1}}{\xi^{\beta+1}(1+\xi x)}+(-1)^{\beta+1} \frac{\beta}{\xi^{\beta+1}} \ln (1+\xi x)-\frac{(-1)^{\beta-1}}{\xi^{\beta+1}} \\
& V(x ; k, \xi, \beta)=\frac{x^{\beta}}{\beta}-\frac{x^{\beta-1}}{\xi(\beta-1)}+\frac{x^{\beta-2}}{\xi^{2}(\beta-2)}-\frac{x^{\beta-3}}{\xi^{3}(\beta-3)}+\ldots-(-1)^{\beta-2} \frac{x}{\xi^{\beta-1}}-(-1)^{\beta-1} \frac{\ln (1+\xi x)}{\xi^{\beta}} .
\end{aligned}
$$


Furthermore,

then, we can obtain

$$
U(x ; k, \xi, \beta)=\int_{0}^{\theta} u^{\beta}(1+\xi u)^{-2} d u,
$$

Suppose,

$$
\begin{aligned}
B_{G}(\theta) & =\int_{0}^{\theta} \frac{4 \xi \exp (\alpha+\beta \ln u)(1+\xi u)^{-2} d u}{1-(1+\xi \theta)^{-1}}, \\
& =\frac{4 \xi \exp (\alpha) U(\theta ; k, \xi, \beta)}{1-(1+\xi \theta)^{-1}} .
\end{aligned}
$$

$$
\begin{aligned}
T(\theta ; k, \xi, \beta) & =\int_{0}^{\theta} \sum_{k=1}^{\beta-1}(-1)^{k-1} \frac{k u^{\beta-k}}{(\beta-k) \xi^{k+1}}+\frac{(-1)^{\beta-1}}{\xi^{\beta+1}(1+\xi u)}+(-1)^{\beta+1} \frac{\beta}{\xi^{\beta+1}} \ln (1+\xi u)-\frac{(-1)^{\beta-1}}{\xi^{\beta+1}} d u \\
& =\sum_{k=1}^{\beta-1} \frac{(-1)^{k-1} k \theta^{\beta-k+1}}{(\beta-k+1)(\beta-k) \xi^{k+1}}+\frac{(-1)^{\beta-1} \beta}{\xi^{\beta+2}} \ln (1+\xi \theta)-\frac{(-1)^{\beta+1} \theta \beta}{\xi^{\beta+1}(1+\xi \theta)}-\frac{(-1)^{\beta-1} \theta}{\xi^{\beta+1}}
\end{aligned}
$$

and let

$$
K(\theta ; k, \xi, \beta)-D(\theta ; k, \xi, \beta)=\int_{0}^{\theta}(1+\xi u)^{-1} U(u ; k, \xi, \beta) d u
$$

Then we get

$$
\begin{gathered}
K(\theta ; k, \xi, \beta)=\sum_{k=1}^{\beta-1} \frac{(-1)^{k-1} k \xi P(\theta ; k, \xi, \beta)}{(\beta-k) \xi^{k+1}} \\
P(\theta ; k, \xi, \beta)=\frac{\theta^{\beta-k}}{\xi(\beta-k)}-\frac{\theta^{\beta-k-1}}{\xi^{2}(\beta-k-1)}+\frac{\theta^{\beta-k-2}}{\xi^{3}(\beta-k-2)}-\ldots+(-1)^{\beta-k-1} \frac{\theta}{\xi^{\beta-k}}+(-1)^{\beta-k} \frac{\ln (1+\xi \theta)}{\xi^{\beta-k+1}} \\
D(\theta ; k, \xi, \beta)=\frac{(-1)^{\beta-1}}{\xi^{\beta+2}}\left((1+\xi \theta)^{-1}-1\right)-(-1)^{\beta+1} \frac{\beta \ln (1+\xi \theta)^{2}}{2 \xi^{\beta+2}}+(-1)^{\beta-1} \frac{\ln (1+\xi \theta)}{\xi^{\beta+2}}
\end{gathered}
$$

Therefore, we have,

$$
\begin{aligned}
\int_{0}^{\theta} G_{X}(u) B_{G}(u) d u & =\int_{0}^{\theta} 4 \xi \exp (\alpha) U(u ; k, \xi, \beta) d u, \\
& =T(\theta ; k, \xi, \beta)-K(\theta ; k, \xi, \beta)+D(\theta ; k, \xi, \beta) .
\end{aligned}
$$

Thus, the weight poverty gap function is given by

$$
\beta_{X^{H}}(\theta)=\frac{\int_{0}^{\theta} G_{X}(u) B_{G}(u) d u}{\theta G_{X}(\theta) B_{G}(\theta)}=\frac{T(\theta ; k, \xi, \beta)-K(\theta ; k, \xi, \beta)+D(\theta ; k, \xi, \beta)}{4 \theta \xi \exp (\alpha) U(\theta ; k, \xi, \beta)} .
$$

Example 4.2 (The Exponential distribution) Drăgulescu and Yakovenko [24] demonstrated that the distribution of individual income in the USA is exponential. Let $Y=\left(y_{1}, y_{2}, \ldots, y_{n}\right)$ be a complete random sample of size $n$ from $\operatorname{Exp}(\lambda)$ as follows:

$$
F_{Y}(\theta)=1-\exp (-\lambda \theta), \quad \theta, \lambda \in \mathfrak{R}^{+} .
$$

Given (4.2), after some algebraic calculations, we obtain

$$
E(4 \exp (\alpha+\beta \ln x) \mid x \leq \theta)=\lambda \exp (\alpha)\left(\frac{\beta !}{\lambda^{\beta+1}}-\exp (-\lambda \theta) \sum_{k=0}^{\beta} \frac{\beta !}{k !} \frac{\theta^{k}}{\lambda^{\beta-k+1}}\right) .
$$

Furthermore, since

$$
\int_{0}^{\theta} u^{\beta-1} \exp (-\lambda u) d u=\frac{(\beta-1) !}{\lambda^{\beta}}-\exp (\lambda \theta) \sum_{k=0}^{\beta-1} \frac{(\beta-1) !}{k !} \frac{\theta^{k}}{\lambda^{\beta-k}} .
$$


Then, we have

$$
\begin{aligned}
A_{F}(\theta) & =\int_{0}^{\theta} \frac{4 \beta \exp (\alpha+\beta \ln u) F_{Y}(u) d u}{u F_{Y}(\theta)} \\
& =\frac{4 \beta \exp (\alpha)}{1-\exp (-\lambda \theta)}\left(\frac{\theta^{\beta}}{\beta}-\left(\frac{(\beta-1) !}{\lambda^{\beta}}-\exp (\lambda \theta) \sum_{k=0}^{\beta-1} \frac{(\beta-1) !}{k !} \frac{\theta^{k}}{\lambda^{\beta-k}}\right)\right)
\end{aligned}
$$

In addition,

$$
\begin{aligned}
B_{F}(\theta) & =\int_{0}^{\theta} \frac{4 \exp (\alpha+\beta \ln u) d F_{Y}(u)}{F_{Y}(\theta)} . \\
& =\frac{4 \lambda \exp (\alpha)}{1-\exp (-\lambda \theta)}\left(\frac{\beta !}{\lambda^{\beta+1}}-\exp (\lambda \theta) \sum_{k=0}^{\beta} \frac{\beta !}{k !} \frac{\theta^{k}}{\lambda^{\beta-k+1}}\right) .
\end{aligned}
$$

Directly by using Eq. (4.6) and Eq. (4.7), we can conclude that

$$
\int_{0}^{\theta} F_{Y}(u) B_{F}(u) d u=4 \lambda \exp (\alpha)\left(\frac{\theta \beta !}{\lambda^{\beta+1}}-\sum_{l=0}^{\beta} \frac{\beta !}{l ! \lambda^{\beta-l+1}}\left(\frac{l !}{\lambda^{l+1}}-\exp (\lambda \theta) \sum_{j=0}^{l} \frac{l !}{j !} \frac{\theta^{j}}{\lambda^{l-j+1}}\right)\right) .
$$

Thus, the weight poverty gap function is given by

$$
\begin{aligned}
& \beta_{X^{H}}(\theta)=\frac{\int_{0}^{\theta} F_{Y}(u) B_{F}(u) d u}{\theta F_{Y}(\theta) B_{F}(\theta)}, \\
& =\frac{\beta !-\sum_{l=0}^{\beta} \frac{\lambda^{l} \beta !}{\theta l !}\left(\frac{l !}{\lambda^{l+1}}-\exp (\lambda \theta) \varphi(\theta ; l, \lambda)\right)}{\beta !-\lambda^{\beta+1} \exp (\lambda \theta) \varphi(\theta ; \beta, \lambda)},
\end{aligned}
$$

where $\varphi(x ; n, \lambda)=\sum_{i=0}^{n} n ! x^{i} /\left(i ! \lambda^{n-i+1}\right)$.

Example 4.3 (The Finite range distribution). Since the finite range distribution have been used to characterize the income between individuals. The cumulative distribution function of the finite range distribution is

$$
\left.F_{X}(\theta)=1-(1-p \theta)^{d}, \quad \theta \in\right] 0, \frac{1}{\mathrm{p}}\left[\text {, and } d, p \in \mathfrak{R}^{+}\right.
$$

Given $\mu$, after some algebraic calculations, we obtain

$$
\begin{aligned}
E(\exp (\alpha+\beta \ln x)) & =\int_{0}^{\theta} p d \exp (\alpha+\beta \ln u)(1-p u)^{d-1} d u, \\
& =p d \exp (\alpha) \frac{\theta^{\beta+1}}{\beta+1}{ }_{2} F_{1}(1-d, \beta+1 ; \beta+2 ; p \theta),
\end{aligned}
$$

whereas ${ }_{2} F_{1}(., . ; . ;$.$) is the generalized hypergeometric function. When$

$$
\int_{0}^{\theta} u^{\beta-1}\left(1-(1-p u)^{d}\right) d u=\frac{\theta^{\beta}}{\beta}\left(1-_{2} F_{1}(1-d, \beta ; \beta+1 ; p \theta)\right),
$$

therefore

$$
\begin{aligned}
A_{F}(\theta) & =\int_{0}^{\theta} \frac{4 \beta \exp (\alpha+\beta \ln u) F_{X}(u) d u}{u F_{X}(\theta)} . \\
& =\frac{4 \beta \exp (\alpha) \theta^{\beta}}{\beta\left(1-(1-p \theta)^{d}\right)}\left(1-{ }_{2} F_{1}(1-d, \beta ; \beta+1 ; p \theta)\right),
\end{aligned}
$$


with the same steps we can conclude that

According to Chaudhry et al. [25], we get

$$
\begin{aligned}
B_{F}(\theta) & =\int_{0}^{\theta} \frac{4 \exp (\alpha+\beta \ln u) d F_{X}(u)}{F_{X}(\theta)} \\
& =\frac{4 d p \exp (\alpha) \theta^{\beta+1}}{(\beta+1)\left(1-(1-p \theta)^{d}\right)}\left(1-{ }_{2} F_{1}(1-d, \beta+1 ; \beta+2 ; p \theta)\right) .
\end{aligned}
$$

$$
\begin{aligned}
\int_{0}^{\theta} F_{Y}(s) B_{F}(s) d s & =4 d p \exp (\alpha) \int_{0}^{\theta} \frac{s^{\beta+1}}{\beta+1}{ }_{2} F_{1}(1-d, \beta+1 ; \beta+2 ; p s) d s, \\
& =4 d p \exp (\alpha) \sum_{n=0}^{\infty} \frac{(1-d)_{n}(\beta+1)_{n}}{(\beta+2)_{n}} \int_{0}^{\theta} \frac{p^{n} u^{n+\beta+1}}{n !} d u, \\
& =\frac{4 d p \exp (\alpha)}{\beta+1} \sum_{l=0}^{\infty} \frac{(1-d)_{l}(\beta+1)_{l}}{(\beta+2)_{l}} \frac{p^{l} \theta^{l+\beta+2}}{(l+\beta+2) l !},
\end{aligned}
$$

where $(Q)_{n}=Q(Q+1)(Q+2) \ldots(Q+n-1)$. Therefore, the weight poverty gap function is given by

$$
\begin{aligned}
\beta_{X^{H}}(\theta) & =\frac{\int_{0}^{\theta} F_{Y}(u) B_{F}(u) d u}{\theta F_{Y}(\theta) B_{F}(\theta)}, \\
& =\frac{\sum_{l=0}^{\infty} \frac{(1-d)_{l}(\beta+1)_{l}}{(\beta+2)_{l}} \frac{p^{l} \theta^{l+\beta+2}}{(l+\beta+2) l !}}{\theta^{\beta+2}{ }_{2} F_{1}(1-d, \beta+1 ; \beta+2 ; p \theta)} .
\end{aligned}
$$

To compare the weighted $P G$ functions of two group poor people, let $X \geq 0$ and $Y \geq 0$ be two random variables (incomes of two poor groups), with density function $k$ and $m$, distribution function $K($.$) and M($.$) ,$ and the weighted PG functions $\beta_{X^{H}}(\theta)$ and $\beta_{Y^{H}}(\theta)$, respectively. The following is the definition of PG orderings. Then $X^{H}$ is said to be $\leq$ to $Y^{H}$ in the poverty gap ordering $\left(X^{H} \leq_{p g} Y^{H}\right)$ if

$$
\beta_{X^{H}}(\theta) \geq \beta_{Y^{H}}(\theta), \quad \text { for all } \theta \in \mathfrak{R}^{+},
$$

where $\theta$ is a poverty line.

It can be written as

$$
\frac{K(\theta) B_{K}(\theta)}{M(\theta) B_{M}(\theta)} \leq \frac{\int_{0}^{\theta} K(s) B_{K}(s) d s}{\int_{0}^{\theta} M(s) B_{M}(s) d s},
$$

whereas

$$
B_{K}(x)=\int_{0}^{x} \frac{H_{K}(u) k(u) d u}{K(x)} \text { and } B_{M}(x)=\int_{0}^{x} \frac{H_{M}(u) m(u) d u}{M(x)} .
$$

In next theorem, we can show that if $X \leq_{p g} Y$, we obtain $X^{H}$ is less than or equal to $Y^{H}$ in $P G$.

Theorem 4.1: Let $X^{H}$ and $Y^{H}$ be two weighted random variables with CDF functions $K^{H}($.$) and D^{H}($.$) ,$ respectively, and $E\left[H_{K}(X)\right]$ and $E\left[H_{D}(Y)\right]$ are exists. Then if $H$ is decreasing in $x$, we get

$$
X \leq_{p g} Y \Rightarrow X^{H} \leq_{p g} Y^{H} .
$$

Proof: Let $X^{H} \leq_{p g} Y^{H}$, then we can get 


$$
\int_{0}^{\theta} K^{H}(u) d u / \int_{0}^{\theta} D^{H}(u) d u d u, \quad \text { is increasing for all } \theta \in \mathfrak{R}^{+} .
$$

since

$$
K^{H}(s)=\frac{K(s)\left[H_{K}(s)-M_{K}(s)\right]}{E\left(H_{K}(s) s \leq \theta\right)} \text { and } D^{H}(s)=\frac{D(s)\left[H_{D}(s)-M_{D}(s)\right]}{E\left(H_{D}(s) \mid s \leq \theta\right)}
$$

where

$$
M_{K}(\theta)=\int_{0}^{\theta} \frac{H_{K}^{\backslash}(u) K(u) d u}{K(\theta)} \text { and } M_{D}(\theta)=\int_{0}^{\theta} \frac{H_{D}^{\backslash}(u) D(u) d u}{D(\theta)} .
$$

Again we rewritten (4.8) as

In addition, (4.8) is occur when

$$
\frac{\int_{0}^{\theta} K(s)\left[H_{K}(s)-M_{K}(s)\right] d s}{\int_{0}^{\theta} D(s)\left[H_{D}(s)-M_{D}(s)\right] d s}, \quad \text { is increasing for all } \theta \in \mathfrak{R}^{+} .
$$

$$
\frac{\int_{0}^{\theta} H^{\backslash}(s) D(s) d s}{\int_{0}^{\theta} H^{\backslash}(s) K(s) d s} \leq \frac{D(\theta)}{K(\theta)} .
$$

where $H_{K}^{\backslash}(x)=\partial H_{K}(x) / \partial x$ and $H_{D}^{\backslash}(x)=\partial H_{D}(x) / \partial x$, since $H$ is decreasing in $s$ and $X \leq_{p g} Y$. The required result follows.

lemma 4.1: Let $H_{F}(x)$ be increasing (decreasing) in $\theta$. Then

1. $B_{F}(\theta) \leq(\geq) H_{F}(\theta)$,

2. $B_{F}(\theta)$ is increasing (decreasing) in $\theta$.

Remark 4.2: If $B_{F}(\theta)$ is replaced by $A_{F}(\theta)$, then lemma 4.1(ii) still holds, but lemma 4.1(i) holds with inequality reversed (cf. Nanda and Jain [26]).

The following theorem shows that for $H_{F}(x)$ increasing in $x$, the weighted random variables and the original random variable are ordered with respect to $\mathrm{RH}$ ordering.

Theorem 4.2: Let $H_{F}(\theta)$ be increasing in $\theta$. Then $X \leq_{r p} X^{H}$.

Proof: From (4.4) we have

$$
F^{H}(\theta) / F(\theta)=B_{F}(\theta) / E\left[H_{F}(\theta)\right],
$$

which is increasing by lemma 4.1(ii). It gives the required result.

Theorem 4.3: The relationship between RPF and the weighted reversed proportional failure rate can be expressed as

Proof: Note that

$$
\widetilde{p}_{X}(\theta)=\frac{\widetilde{p}_{X^{H}}(\theta)}{H_{F}(\theta)}\left[\int_{0}^{\theta} \frac{\widetilde{p}_{X^{H}}(u)}{H_{F}(u)} \exp \left(-\int_{u}^{\theta} \widetilde{p}_{X^{H}}(y) d y\right) d u\right]^{-1} .
$$


Further, since

$$
\begin{aligned}
& \widetilde{p}_{X}(\theta)=\frac{\theta E\left[H_{F}(\theta)\right] f^{H}(\theta) / H_{F}(\theta)}{\int_{0}^{\theta} E\left[H_{F}(u)\right] f^{H}(u) / H_{F}(u) d u} \\
& =\frac{\widetilde{p}_{X^{H}}(\theta)}{H_{F}(\theta)} / \int_{0}^{\theta} \frac{\widetilde{p}_{X^{H}}(u) F^{H}(u)}{H_{F}(u) F^{H}(\theta)} d u .
\end{aligned}
$$

We have

$$
F^{H}(\theta)=\exp \left(-\int_{\theta}^{\infty} \widetilde{p}_{X^{H}}(u) d u\right)
$$

$$
\frac{F^{H}(t)}{F^{H}(\theta)}=\exp \left(-\int_{t}^{\theta} \tilde{p}_{X^{H}}(u) d u\right),
$$

then by (4.9), and (4.10) gives the required result. $\square$

The next result studies the relationship between the $\beta_{U^{H}}(\theta)$ and $\beta_{U}(\theta)$ by using the variance of the poverty gap as follows:

Theorem 4.4: Let $U \geq 0$ be a random variable with distribution function $F$. Therefore, the weighted poverty gap function can be expressed as

$$
\beta_{U^{H}}(\theta)=\beta_{U}(\theta)-\left(\sigma_{U}^{2}(\theta) /\left(\theta-\beta_{U}(\theta)\right)\right),
$$

where $\sigma_{U}^{2}(\theta)$ is variance of the poverty gap.

Proof: Since

$$
\beta_{U^{H}}(\theta)=\int_{0}^{\theta}\left(u-\beta_{U}(u)\right) F(u) d u /\left(F(\theta) /\left(\theta-\beta_{U}(\theta)\right)\right),
$$

and

$$
\int_{0}^{\theta} \beta_{U}(s) F(s) d s=\int_{0}^{\theta}(1-s) F(s) d s / \theta
$$

Then, we can get that

$$
\sigma_{U}^{2}(\theta)=\frac{1}{\theta^{2}}\left(2 \theta \beta_{U}(\theta)-\beta_{U}^{2}(\theta)-\int_{0}^{\theta} 2 t F(t) d t / F(\theta)\right) .
$$

Then, the required result follows.

Theorem 4.5: Let $X \geq 0$ be a random variable with absolutely continues distribution function $F$, then the weighted variance of the poverty gap function can be expressed as

$$
\sigma_{X^{H}}^{2}(\theta)=\left(\left(\int_{0}^{\theta} \int_{0}^{u} 2 B_{F}(t) F(t) d t d u / B_{F}(\theta) F(\theta)\right)-\beta_{X}^{2}(\theta)\right) / \theta^{2} .
$$

Proof: The proof follows on using (4.5) and by noting the fact that

$$
\sigma_{X^{H}}^{2}(\theta)=\left(\left(\int_{0}^{\theta} \int_{0}^{u} 2 F^{H}(t) d t d u / F^{H}(\theta)\right)-\beta_{X^{H}}^{2}(\theta)\right) / \theta^{2} . \square
$$

The importance and the role of power distribution in basic income studies is introduced by Dimaki and Xekalaki [27]. In the next example, we characterization $\leq_{p g}$ for power distribution based on a relationship between $\beta_{X}(\theta)$ and $\beta_{X^{H}}(\theta)$.

Example 4.4: Let $Y=\left(y_{1}, y_{2}, \ldots, y_{n}\right)$ be a complete random sample of size $\mathrm{n}$ from a power distribution with $\mathrm{CDF}$ as follows

$$
F_{Y}(y)=(y / \kappa)^{\varsigma}, \quad 0<y<\kappa ; \kappa, \varsigma \in \mathfrak{R}^{+} .
$$


and $Y^{H}$ is weighted random variable of $\mathrm{Y}$ with weighted function $H(y)=4 \exp (\alpha+\beta \ln y)$. Then, we can get

$$
\beta_{F}(\theta)=\int_{0}^{\theta}(x / \kappa)^{\varsigma} d x /\left(\theta(\theta / \kappa)^{\varsigma}\right)=1 /(\varsigma+1)
$$

Moreover,

and

$$
E[\exp (\alpha+\beta \ln \theta)]=4 \exp (\alpha) \varsigma(1 / \kappa)^{\varsigma} \theta^{\beta+\varsigma} /(\beta+\varsigma)
$$

In addition, by using Eq. (4.5), we have

$$
B_{F}(\theta)=4 \exp (\alpha) \varsigma \theta^{\beta} /(\beta+\varsigma)
$$

Then, we can conclude that,

$$
\beta_{Y^{H}}(\theta)=1 /(\beta+\varsigma+1),
$$

$$
\begin{cases}Y<_{p g} Y^{H}, & \forall \beta>0, \\ Y \geq_{p g} Y^{H}, & \forall \beta \leq 0 .\end{cases}
$$

Example 4.5: Let $U \geq 0$ and $V \geq 0$ be two continuous random variables, with the distribution functions $F_{U}=\left(u / \kappa_{1}\right)^{\varsigma_{1}}, \quad 0<u<\kappa_{1} ; \kappa_{1}, \varsigma_{1}>0$ and $G_{V}=\left(v / \kappa_{2}\right)^{\varsigma_{2}}, \quad 0<u<\kappa_{2} ; \kappa_{2}, \varsigma_{2}>0$, PG functions $\beta_{F}(\theta)$ and $\beta_{G}(\theta)$, weighted distribution functions $F^{H}(\theta)$ and $G^{H}(\theta)$ and weighted poverty gap functions $\beta_{F^{H}}(\theta)$ and $\beta_{G^{H}}(\theta)$, respectively. We can say:

1. $F \leq G$ in poverty gap ordering (denoted as $U \leq_{p g} V$ ) if $\varsigma_{2} \geq \varsigma_{1}$,

2. $F^{H}(\theta)$ is smaller than $G^{H}(\theta)$ in poverty gap ordering (denoted as $F^{H}(\theta) \leq_{p g} G^{H}(\theta)$ if $\varsigma_{2} \geq \varsigma_{1}$.)

Note that: The comparison between two random variables (weighted random variables) with power distribution depend on power parameter $(\varsigma)$.

\section{Acknowledgements}

The author would like to express her thanks and gratitude to the editor and reviewers for their efforts, great comments, and suggestions that add to more quality of the manuscript

\section{References}

[1] A.B. Atkinson, On the measurement of poverty, Econometrica. 55 (1987) 749-764.

[2] J. E. Foster and A. F. Shorrocks, Poverty orderings, Econometrica, 56 (1988a) 173-178.

[3] J. E. Foster and A. F. Shorrocks, Poverty orderings and welfare dominance, Soc. Choice. Welfare. 5 (1988b) 179-198.

[4] F. Belzunce, J. Candel and J. M. Ruiz, Ordering and asymptotic properties of residual income distributions. Sankhya. Ser B. 60 (2) (1998) 331-348.

[5] A. Sen, Poverty: An Ordinal Approach to Measurement. Econometrica. 44(2) (1976) 219-231

[6] H. W. Block, T. H. Savits and H. Singh, The reversed hazard rate function, Probab. Eng. Inform. Sc. 2 (1998) 69-90.

[7] N. K. Chandra and D. Roy, Some results on reversed hazard rate, Probab. Eng. Inform. Sc. 15 (2001) 95-102.

[8] M. S. Finkelstein, A Note on some aging properties of the accelerated life model, Reliab. Eng. Syst. Safe. 71 (2001) 109-112.

[9] M. S. Finkelstein, On the reversed hazard rate, Reliab. Eng. Syst. Safe. 78 (2002) 71-75.

[10] M. S. Finkelstein, On one class of bivariate distributions, Stat. Probab. Lett. 65 (2003) 1-6.

[11] R. C. Gupta, R. D. Gupta and P. L. Gupta, Monotonicity of the (reversed) hazard rate of the (maximum) minimum in bivariate distributions, Metrika 63 (2006) 223-241.

[12] M. O. Lorenz, Methods of measuring the concentration of wealth, J. Am. Stat. Assoc. 9 (1905) 209-219.

[13] J. L. Gastwirth, A general definition of the Lorenz curve, Econometrica. 39 (1971) 1037-1039.

[14] W. A. Thompson, Fisherman's luck, Biometrics. 32 (1976) 265-271.

[15] B. C. Arnold, Pareto Distributions. (International Co-operative Publishing House, 1983) 
[16] I. A. Ahmad, M. Kayid and F. Pellerey, Further results involving the MIT order and the IMIT class, Probab. Eng. Inf. Sci. 19 (2005) 337-395.

[17] M. Kayid and I. A. Ahmad, On the mean inactivity time ordering with reliability applications, Probab. Eng. Inform. Sc. 18 (2004) 395-409.

[18] G. T. Borjas, The Relationship between wages and weekly hours of work: The role of division Bias, J. Hum. Resour. 15(3) (1980) 409-423

[19] A. Di Crescenzo, Some results on the proportional reversed hazard model, Stat. Probab. Lett. 50 (2000) 313-321

[20] G. Solon, Intergenerational Income Mobility in the United States, Am. Econ. Rev. 82(3) (1992) 326-329.

[21] R. Barrett and M. Salles, On three classes of differentiable inequality measures, Int. Econ. Rev. 39 (1998) 611-621.

[22] A. Ichino, F. Mealli and T. Nannicini, From temporary help jobs to permanent employment: What can we learn from matching estimators and their sensitivity, J. Appl. Econ. 23 (2008) 305-327.

[23] K. S. Lomax, Business failures; another example of the analysis of failure data, J. Am. Stat. Assoc. 49 (1954) 847--852.

[24] A. Drăgulescu and V. M. Yakovenko, Exponential and power-law probability distributions of wealth and income in the United Kingdom and the United States, Physica A. 299 (2001) 213--221

[25] M.A. Chaudhry, A. Qadir, H.M. Srivastava, R.B. Paris, Extended hypergeometric and confluent hypergeometric functions, Appl. Math. Comput. 159, (2004) 589-602

[26] A. Nanda and K. Jain, Some weighted distribution result on univariate and bivariate cases, J. Stat. Plan. Inference 77 (1999) 169 180.

[27] C. Dimaki and E. Xekalaki, Identifiability of Income Distributions in the Context of Damage and Generating Models, Commun. Stat-Theor. M. 19(8) (1990) 2757-2766 